\title{
Considerations in Head and Neck Oncologic Reconstructions and Microsurgery During COVID-19 Pandemic
}

\author{
Dr. Suvashis Dash, AIIMS, New Delhi, India ${ }^{1,2,3,4,5}$ and Dr. Vinay Kant Shankhdhar ${ }^{6}$ \\ ${ }^{1}$ COVID vs Cancer: Impact on Head and Neck Oncology \\ ${ }^{2}$ Dr. Shivangi Saha, AIIMS, New Delhi, India \\ ${ }^{3}$ Dr. Maneesh Singha, Head of Department, Plastic Surgery, AIIMS, New Delhi, India \\ ${ }^{4}$ Dr.Shivakumar Thiagarajan, Tata Memorial Hospital, HBNI, Mumbai, India \\ ${ }^{5}$ Dr. Pankaj Chaturvedi, Tata Memorial Hospital, HBNI, Mumbai, India \\ ${ }^{6}$ Professor and Head, Plastic Surgery, Tata Memorial Centre, HBNI, Mumbai, India.
}

May 11, 2020

\begin{abstract}
COVID outbreak has incapacitated the healthcare system around the world. Existing resources and manpower are being redirected to take care of the COVID-19 disease patients. People with head and neck cancers with the need of post ablative reconstruction are in difficult situation owing to multiple factors like poor general condition, disease progression and potential chance of getting an infection of COVID -19 in a health care facility as well as doubt regarding treatment completion i.e. adjuvant treatment. Appropriate reconstruction following ablative surgery, especially in advanced disease, facilitates functional recovery and thus adding to the quality of life of the patients. The reconstructive procedures are resource-intensive, requiring long hours of surgery, trained manpower, and multiple team members. However, if adequate surgical excision demands the reconstructive procedure, then it should not be a hindrance for the standard treatment. We need to review our approach in the face of the devastating COVID-19 pandemic. We are presently working in resource constraints like limited availability of staff and limited availability of personal protective equipment especially in plastic surgery procedures which requires the use of loupes and microscope. Thus, the challenge is to ensure proper reconstruction with limited available resources and maintaining safety standards for the staff in the operation theatre. This work is based on our experience and evidence from the literature.
\end{abstract}

Keywords- oncologic reconstruction; Microsurgery; COVID-19; Head neck cancer; reconstructive surgery; Head neck reconstruction.

\section{Background}

COVID-19 pandemic has changed many aspects of our lives in the past few months. This disease has started from Hubei provenience of China in 2019, December and eventually it became a global pandemic by March 2020 involving more than 214 countries $^{1}$. During the time of drafting $(24 / 04 / 2020)$ as per the global data, around 27 million people have been tested positive, while more than 188,000 succumbed to the disease ${ }^{2}$. This is primarily a respiratory tract infection transmitted by human to human contact, respiratory droplets and aerosols. ${ }^{3}$

The epidemiology of head and neck cancer varies among geographical regions, worldwide yearly more than 650,000 cases of head and neck cancers are detected and 330,000 deaths are attributed to it ${ }^{4}$. Asia has around $57 \%$ of the global burden of head and neck cancer patients as well as the maximum number of 
deaths ${ }^{5}$. It was projected that by year2020, South-east Asia will have a steep increase (75\%) in the number of cancer deaths. ${ }^{6}$

Due to the high rate of infectivity, Health care workers are faced with a daunting task, while performing duty in the time this pandemic. Recently, some recommendations and protocols have been put forward by various researchers to deal with practices in head and neck surgeries, plastic surgery but points in postcancer reconstruction need to be discussed. ${ }^{7,8}$ this article aims to discuss available evidence and provide key suggestions, specifically for head and neck onco-reconstruction and microvascular surgery, as well as offer guidelines to enhance infection control, patient safety, and resource management. Do we compromise the quality of reconstruction or adopt certain changes to suit the present scenario?

\section{Potential challenges}

In the face of the current pandemic, the cancer surgeons are at times forced to delay excision but the balance between the harm of delaying the surgery and protection from infection is hard to assess in general ${ }^{9}$. Cancer is considered as a semi-emergency and delaying treatment is difficult both for the treating oncologist and the patients. The pandemic in India at present may be at best described as early and its number of new cases of COVID-19 would only increase in the coming days despite the present lockdown. Cancer patients are exposed to the dual risk of cancer and COVID infection. Although, the initial reports suggest grave outcomes in cancer patients ${ }^{10}$. The evidence is inadequate for any conclusion. The onco-reconstructions have moved a long way from simple defect cover to better quality approach with more functional and aesthetically pleasing reconstructions ${ }^{11}$. Considering the present setting, it may be correct to predict that, the quality and quantity of reconstructions may be compromised, for at least some time. Just to highlight the demands in India, Tata Memorial Centre, a premier cancer centre in Mumbai, alone handles yearly load of approximately 900 cancer reconstructions in the department of Plastic and Reconstructive Surgery of which more than $60 \%$ are free flaps.

- During the pandemic resource allocation for the cancer reconstruction may not be optimal.

- The general condition of cancer patients may pose a hindrance to the time-consuming reconstructive procedures.

- In the postoperative period: intensive care, availability of mechanical ventilators may be problematic in the pandemic, considering the resource crunch.

- Cancer patients as such are considered immunocompromised and the addition of chemotherapy and/or radiotherapy may only lower their immunity further and increase the risk of mortality due to COVID$19 .{ }^{12}$

- Providing adequate reconstruction while maintaining the standard of multi-disciplinary care will be tough in the scenario.

- Due to the pandemic there may be a reduced number of operation theaters functioning and available manpower.

The overall plan of an onco-reconstruction is dependent on the capacity of the setup, capability and skill level of the team, availability of equipment, adequate infrastructure, and the aesthetic and functional requirement of the patients. (Fig 1) 


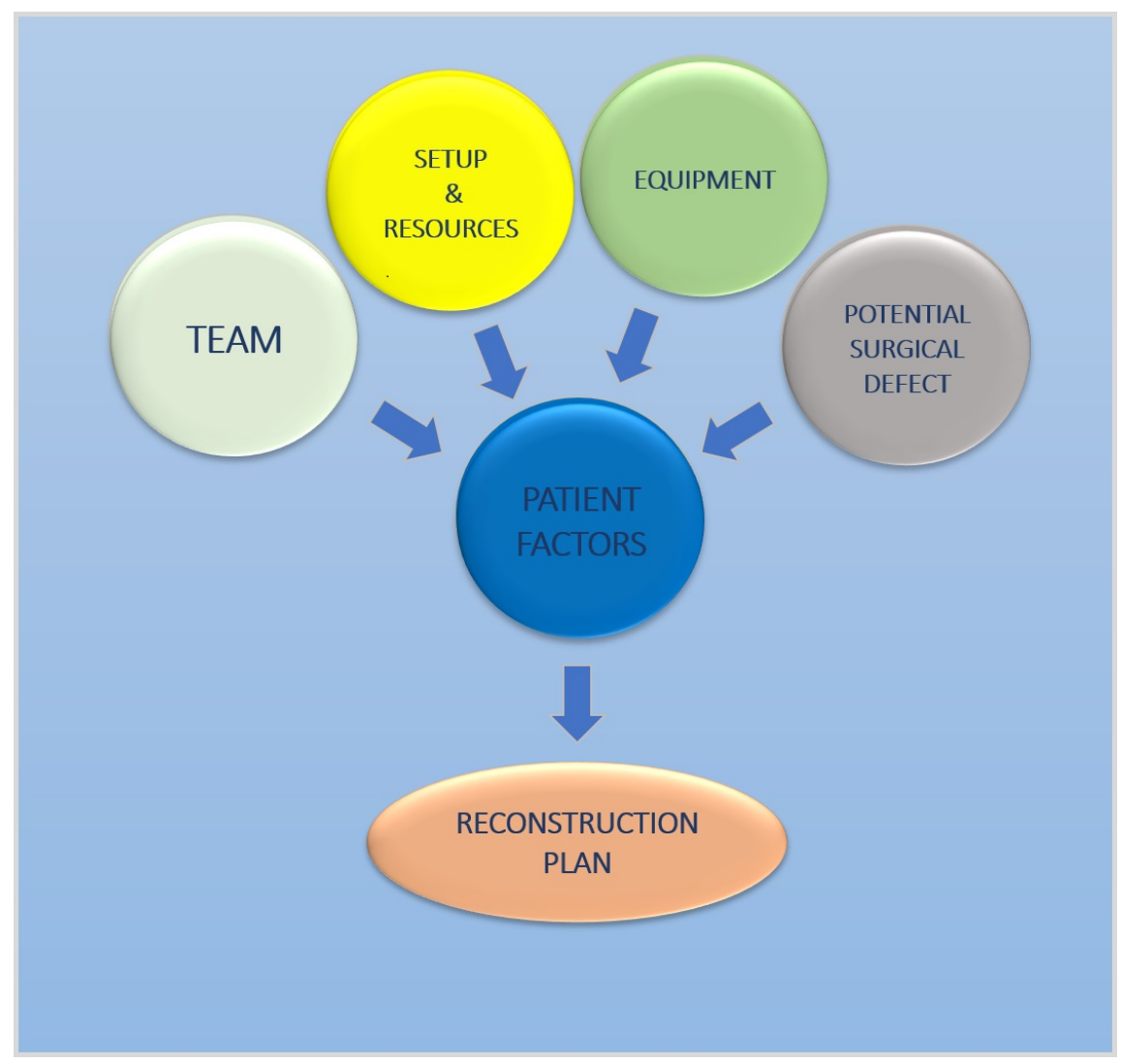

Figure 1: Factors affecting the reconstruction plan

\section{The setup}

Most of the large health centers are now overwhelmed by patients of COVID-19. The non-COVID-19 health care set up is also at risk of cross-contamination due to rampant community infection. Patients with history relating to FTOCC (fever, travel, occupation, contact, and clustering) mandates Screening ${ }^{13}$. Many hospitals use pre-operative COVID screening, though the hospital policies may differ from place to place. All asymptomatic patients should be considered as potential infective cases and all precautions should be taken while managing them. If a patient planned for surgery is positive, the surgery should be postponed till he/she becomes negative. The emergency surgeries like carotid blowouts, acute bleeds, etc. however may need intervention even inactive COVID infection, such cases would need surgeries with full PPEs and in designated COVID operation theatres. In the future, the pre-operative viral test may become mandatory.

Isolation facility for high-risk patients may be done to prevent the spread of infection. The areas can be marked as contaminated, non-contaminated, and potentially contaminated areas to stratify the risk. Route and lifts of these areas should be separate to prevent cross-contamination. Biomedical waste handing consideration as per standard protocol should be done ${ }^{13,14}$. The significant point to contemplate is whether the hospital is treating both COVID and non-COVID patients or is it a specialized center for cancer treatment. The role of tertiary centers and specialized cancer hospitals, which does not deal with COVID-19 patients directly have a vital role for onco-reconstruction. It is understandable that, due to some patients and the health care staff developing COVID infections and thereby resulting in compromise of the resources, the overall number of cancer surgeries and reconstructive procedures may decrease. Even minor reconstruction for oncological defects can be carried out in smaller hospitals to decrease the burden on major hospitals. It is wise to think of both the COVID case burden in community patients and hospital capacity before planning 
the protocol for management.

\section{The team members}

The reconstructive team comprises plastic surgeons, anesthesiologists, nurses, physiotherapists, and support staff. Roster for dedicated teams comprising of different members of the team should be made on rotation as per the availability. Due to the prolonged nature of the surgeries in head and neck onco-reconstruction compared to the other surgical procedures, the risk of infection and cross infection is possibly more. The reconstructive scenario involves a multiteam approach, such as a team for flap harvest and team for vessel preparation, which leads to overcrowding of the operative room. Provisions should be made to reduce overcrowding. We must consider the safety of the health care workers as the prime concern.

The following points need mention

1. The team should undergo Training in the proper use of Personal protective equipment (PPE), Sequence of donning and doffing.

2. Every health care worker to use the face shield, N95 mask while working to protect the splashing of body fluid to eyes, face ${ }^{13,14}$.

3. The face shields for plastic surgeons should be deep enough to accommodate the loupes.

4. Bone cutting, drills, and power tools- these cause more aerosol generation, these aerosols may be a source of infection- should be avoided or done with precaution. ${ }^{16,17,18} \mathrm{~A}$ adhesive transparent sheet may be used to cover the saw and drill to prevent splashing in different directions. The irrigation and cutting/drilling should alternate to avoid splashing and minimize aerosol generation.

5. During aerosol generation procedures like mandibular excision, fibular osteotomies, and tracheostomies, etc. only the minimum number of team members are allowed in the operation theatre. The ancillary staff may be asked to remain out of the operation theatre and the remaining surgeons not directly involved in the procedure may stand as far as possible (since two teams are simultaneously working).

6. The anastomosis may be under loupes using a deep face shield that accommodates the loupes or

7. Anastomosis under the microscope - use of face shield is not possible as the focusing is difficult due to the increased distance between the operating surgeon's eye and microscope eyepiece. Hence, the use of protective safety glasses may be very useful to cover the eyes.

8. The tracheostomy is covered using adhesive plastic cover (to ensure avoidance of air blast in accidental disconnection of the tracheostomy tube).

9. All the procedures are done by experienced surgeons to reduce the time of surgery and reduce the chances of flap failure, re-explorations, and any untoward incidents in times of limited resources and manpower.

\section{Equipment and inventory}

The SARS-CoV-2 is a highly infective pathogen. It is imperative that the use of equipment during COVID-19 has a chance of becoming a source of cross-contamination and spread the disease to noninfected individuals and health care workers. Isolation of the instruments to be used for the COVID-19 patients is necessary ${ }^{15}$. Specifically, at least two sets of micros instruments are needed for the smooth functioning of reconstructive surgery. Cleaning of the noncritical equipment with $1 \%$ hypochlorite solution. ${ }^{16}$

\section{Prioritization of cases}

The cases of reconstruction should be discussed with the team in respect to administrative, clinical and nursing concerns. If adequate manpower is not available transferring the patient to another center is essential. Patients who will benefit most from the reconstruction should be considered. Cases should be triaged based on Clinical features and tumor behavior, the outcome of surgery and a person's general condition, comorbidities, and availability of resources. ${ }^{8,19}$

\section{Special groups}

High-risk groups constitute Elderly individuals, patients with Comorbid conditions, patients undergoing 
chemotherapy and/or radiotherapy, and patients with immune modulators. ${ }^{20}$ This group needs special care and isolation. It is important to remember, that reconstructive surgery will allow the person to undergo radiotherapy earlier.

\section{Follow-ups, physiotherapy}

After reconstructive surgeries, patients need to follow up visits, re-surgery, and physiotherapy for functional and aesthetic improvement. It is better to understand the individual needs of the patients. These can be segregated into essential and non- essential visits by the use of teleconsultation. The risk-benefit ratio should be compared for the patients. Patients should only visit for necessary interventions. Follow up using video calls and mobile application should be encouraged. ${ }^{21}$

\section{Suggestion for different scenarios}

\author{
Reconstruction type \\ Local flaps, Skin grafts $<$ \\ $50 \mathrm{~cm}^{2}$ \\ Regional flaps,Skin grafts \\ for large areas \\ Free flaps / Extensive \\ reconstruction with \\ multiple flap surgery \\ Exploration of free flaps, \\ Flap failure conditions
}

Factors (Region, tumor clearance, Comorbidities)
\pm Comorbidities, R0 resection possible
\pm Comorbidities, R0-resection, Good general condition of the patient.
\pm Comorbidities, doubtful tumor clearance, poor general condition of the patient.

\pm Comorbidities, \pm tumor clearance, \pm general condition of the patient.
Recommendation

Proceed with the surgery.

Proceed with the surgery with adequate precautions.

If an alternative procedure can give adequate results, change plan to lesser invasive procedure.

Proceed with the surgery with adequate precautions, reexploration / salvage flap

Table 1: Suggestion for different scenarios oncologic reconstruction and Microsurgery during the time of COVID-19 pandemic

\section{Discussion with onco-surgeon}

If the tumor needs excision then reconstructive procedure should not be a limitation for completion of treatment. While certain rational decisions for resection of tumor merit attention:-

- Only non-emergency or secondary reconstructions may be postponed. Cancer surgery should not be postponed. ${ }^{22}$

- Non-surgical alternative maybe be considered if they give comparable results. ${ }^{23}$

- Consent should include the risk of COVID-19 infection, associated burden of disease, and points covering reconstructive procedures. It should also be covered by the reconstructive team. ${ }^{9}$

- Case with uncertain tumor clearance or palliative surgery - nonsurgical method of treatment to be encouraged.

- Avoidance of tracheostomy if possible. If tracheostomy performed small size stoma should be done, small cuffed, nonfenestrated tube should be used ${ }^{18,24}$

- Avoidance of rotatory tools, which increase the aerosol generations. ${ }^{18}$

\section{Surgical factors}

\section{The head and neck reconstruction and microvascular surgery}


In the head and neck region the reconstructive surgeries have more risk compared to other regions of the body. COVID-19 disease primarily involves the respiratory tract. Respiratory droplets and oral secretion may cause infection and cross infection in the surroundings. Care in the reconstructive procedures to reduce aerosol generation. ${ }^{18}$ During the time of patient induction and extubation, minimal personal should be present in the room. Similarly, at the time of tracheostomy also nonessential persons should be out of the room. If possible, tracheostomy to be done under the transparent plastic sheet to minimize the spread of droplets.

\section{Flap selection, harvest and anastomosis}

Pedicled or free flap! what should be the choice of reconstruction? This question comes to the mind of a reconstructive surgeon. Again, this will depend upon the resources, availability of operating rooms, time, and the defect. Both free and pedicle flaps have their roles, usually, the workhorse flaps like Free fibula, free anterolateral thigh flap, free radial artery forearm flap, and Latissimus dorsi should be done as a routine. Some cases of lateral mandibular defect can be reconstructed with a pectoralis major flap instead of free flaps. The deltopectoral flap should be avoided for perioral defects, as continuous drooling of oral secretions may occur. In cases of mandibular reconstruction with the fibula, putting primary dental implants can be avoided.

To decrease the operative time, the flap should be preferentially harvested by the senior surgeons. This will also decrease the error rate of the procedure. Some reports have suggested to reduce intraoperative surgical smoke, as it can be a potential source of infection. Hence special consideration like, using bipolar electrocautery favorably, or monopolar electrocautery with low power settings to reduce surgical smoke, use of suture materials and clips is encouraged. Care should be given to adequate smoke dispersal in the operating room ${ }^{25}$. The most experienced surgeon must do the critical steps of surgery, like harvest and vascular anastomosis to get optimal success ${ }^{18}$. During anastomoses near the head and neck region putting a transparent adhesive surgical drape to cover the aerodigestive tract will provide some protection. While operating an N95 mask or powered air-purifying respirator (PAPR) is a must.

A generalized plan for the type of reconstruction, will depend on the extent of oncological clearance, the general condition of the patient, and the presence of comorbidities (Table 1). A region-wise consideration may depend on the risk vs benefits of the reconstruction and an individualized clinical judgment on a case to case basis.

\section{Microscope, loupe, and light source.}

In reconstructive microsurgery the operating microscope and loupes are valuable tools, it is difficult to use PPE, goggles, and face shield due to improper fitting, repeated fogging, and extra weight. Face shields that have adequate space to accommodate the surgical loupes can be used or Making customized holes in the face shield for proper airtight fitting of loupes along with the light source will be helpful. Disinfection of a microscope is a concern and should be done with care. It is best to cover the microscope in the recommended company provided covers. Anastomosis of medium to large caliber vessel if possible, to perform comfortably under a loupe with shield, should be done. In the case of smaller caliber vessels, when an operating Microscope is required, then use protective goggles, N95 Mask along with microscope. The operating area is covered with adhesive sheets.

\section{Flap monitoring, pack removals, Drains, Tubes and Wound Dressing}

During flap monitoring and pack removals care must be taken as body fluids from flap margin are potentially infective. It is critical to understand that, wound dressing, tube, and drain removals are to be done with utmost care and following proper protective protocols. Use of face shields, N95 masks, and gloves are advised for all dressings and ward rounds.

\section{Flap exploration}

This is important to consider that during the pandemic time the exploration of the flap should proceed as 
routine, but the most experienced surgeon should perform it with all precautions.

\section{Non-surgical factors}

Some factors which are nonsurgical but are important to consider during these times. Due to restrictions in the transportation across the country, people from far-off places may not be able to attend frequent follow-ups. teleconsultation facilities should be utilized to benefit such patients.

Proposed Reconstruction Algorithm for Various Defects in Head and Neck Cancer during COVID pandemic

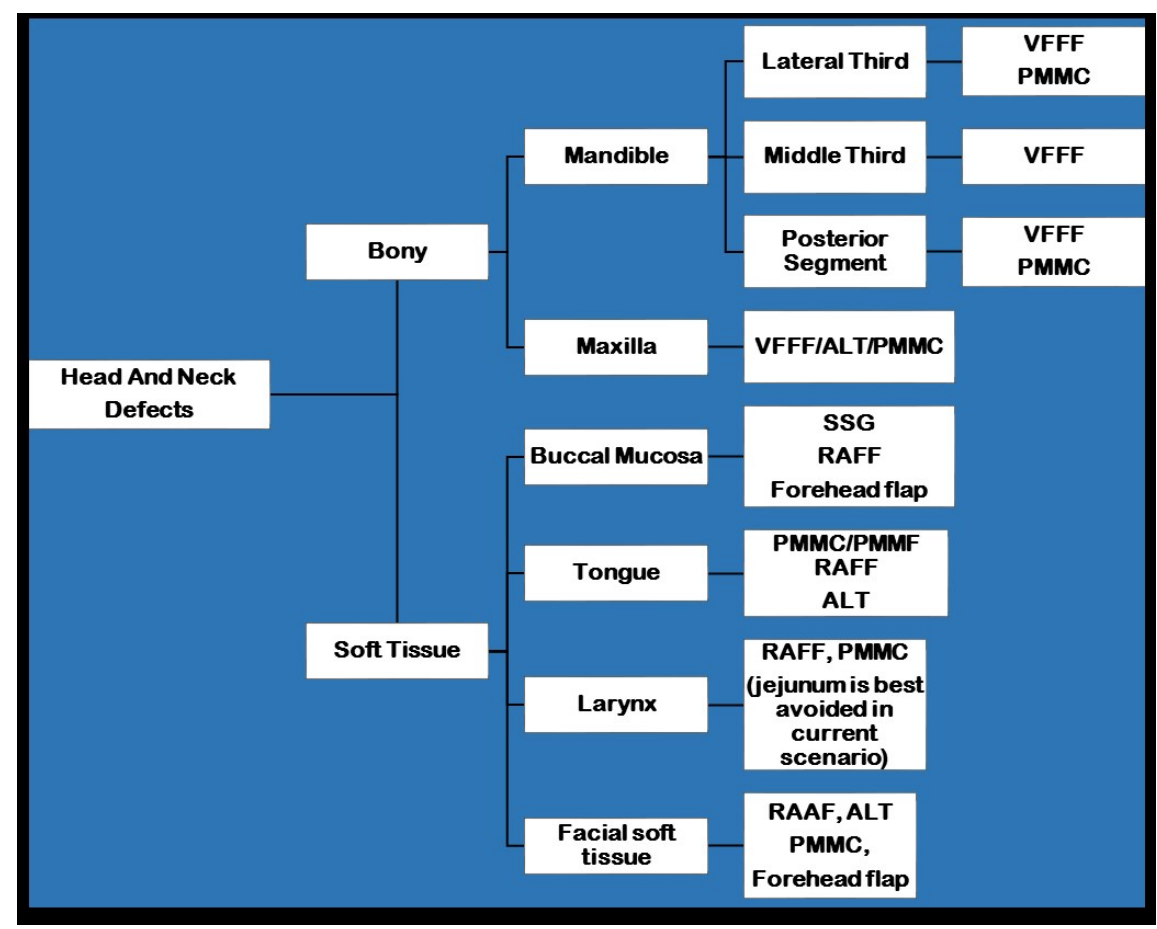

ALT- (Free) Anterolateral Thigh Flap, PMMC- (Pedicled)Pectoralis Major Myocutaneous Flap, PMMF-(Pedicled)Pectoralis Major Myofascial Flap, ALT- Free Anterolateral Thigh Flap, RAFF- (Free) Radial Artery Forearm Flap, VFFF- Vascularized Free Fibula Flap

\section{Conclusions}

Cancer is a disease that cannot be deferred for long, and for adequate surgical treatment, reconstruction is an absolute necessity. Centers dedicatedly dealing with the cancer treatment are important in this time to provide as much cancer care as possible as general hospitals may not be available. The need is to avoid the increase in the deaths that may result due to deficient cancer care in the face of this pandemic. Reconstruction should not be a limitation for head and neck cancer treatment. Reconstruction should be carried out in discussion with the team, considering the capacity of setup, adequacy of resources, equipment, and potential defect. Isolation of high-risk patients, risk stratification of patients, infection control, and protection of health care workers are important considerations during these times. The use of technology via online platforms comes handy for follow-ups and consultation.

\section{Key points}


- Cancer is considered as a semi-emergency and cancer treatment should be triaged rather than deferring, during the COVID-19 pandemic.

- Cancer reconstruction is vital for the adequacy of overall treatment in many ways, and it poses lots of challenges and ethical dilemmas.

- Reconstruction should not be the limiting factor for adequate cancer treatment.

- A disclaimer covering the associated risk of COVID-19 with malignancy as well as points covering reconstructive procedure is vital and to be noted in the consent.

- Dedicated cancer care centers should lead the way for reconstructive care in these times.

- Team, setup, resources, and potential defects are important considerations.

- Infection control and the protection of health care workers should be a priority

\section{References}

1. WHO announces COVID-19 outbreak a pandemic available at http://www.euro.who.int/ en/health-topics/health-emergencies/coronavirus-COVID-19/news/news/2020/3/whoannounces-COVID-19-outbreak-a-pandemic (Accessed on 24th April,2020)

2. COVID-19 coronavirus pandemic available at https://www. worldometers.info/coronavirus/ (Accessed on $24^{\text {th }}$ April,2020)

3. Modes of transmission of virus causing COVID-19: implications for IPC precaution recommendation available at https://www.who.int/news-room/commentaries/detail/modes-of-transmissionof-virus-causing-covid-19-implications-for-ipc-precaution-recommendations (Accessed on $24^{\text {th }}$ April,2020)

4. Bray F, Ferlay J, Soerjomataram I, et al. Global cancer statistics 2018: GLOBOCAN estimates of incidence and mortality worldwide for 36 cancers in 185 countries. CA Cancer J Clin 2018; 68:394

5. Chaturvedi P. Head and neck surgery. J Can Res Ther 2009; 5:143

6. Mishra A, Meherotra R. Head and Neck Cancer: Global Burden and Regional Trends in India. Asian Pacific Journal of Cancer Prevention [Internet]. 2014 Jan 30;15(2):537-50. Available from: http: //dx.doi.org/10.7314/APJCP. 2014.15.2.537

7. Kowalski LP, Sanabria A, Ridge JA, Ng WT, de Bree R, Rinaldo A, Takes RP, Mäkitie AA, Carvalho AL, Bradford CR, Paleri V. COVID-19 pandemic: effects and evidence-based recommendations for otolaryngology and head and neck surgery practice. Head \& Neck. 2020 Apr 9.

8. COVID 19: Elective Case Triage Guidelines for Surgical Care Available at https://www.facs.org/ /media/files/COVID19/guidance_for_triage_of_nonemergent_surgical_procedures_plastic. ashx (Accessed on $18^{\text {th }}$ April,2020)

9. Brahma B. Oncologists and COVID-19 in Indonesia: What can we learn and must do?. Indonesian Journal of Cancer. 2020 Mar 30;14(1):1-2.

10. Liang W, Guan W, Chen R, Wang W, Li J, Xu K, et al. Cancer patients in SARS-CoV-2 infection: A nationwide analysis in China. Lancet Oncol. 2020;21(3):335-7

11. Hayden RE, Nagel TH. The evolving role of free flaps and pedicled flaps in head and neck reconstruction. Current opinion in otolaryngology \& head and neck surgery. 2013 Aug 1;21(4):305-10.

12. Kang DH, Weaver MT, Park NJ, Smith B, McArdle T, Carpenter J. Significant impairment in immune recovery following cancer treatment. Nursing research. 2009 Mar;58(2):105.

13. Lai TH, Tang EW, Chau SK, Fung KS, Li KK. Stepping up infection control measures in ophthalmology during the novel coronavirus outbreak: an experience from Hong Kong. Graefe's Archive for Clinical and Experimental Ophthalmology. 2020 Mar 3:1-7.

14. Lui RN, Wong SH, Sanchez-Luna SA, Pellino G, Bollipo S, Wong MY, Chiu PW, Sung JJ. Overview of guidance for endoscopy during the coronavirus disease 2019 (COVID-19) pandemic. Journal of Gastroenterology and Hepatology. 2020 Mar 31.

15. Di Saverio S, Pata F, Gallo G, Carrano F, Scorza A, Sileri P, Smart N, Spinelli A, Pellino G. Coronavirus pandemic and Colorectal surgery: practical advice based on the Italian experience. Colorectal Disease. 2020 Mar 31.

16. Information For Healthcare Professional, available at https://www.cdc.gov/coronavirus/2019- 
ncov/healthcare-facilities/index.html (Accessed on $24^{\text {th }}$ April,2020)

17. AO CMF International Task Force Recommendations available at https://www.aofoundation. org/what-we-do/COVID-19-resources-for-surgeons\#COVIDnews_o=News\%20Date\%20Facet, Descending (Accessed on $24^{\text {th }}$ April,2020)

18. Givi B, Schiff BA, Chinn SB, Clayburgh D, Iyer NG, Jalisi S, Moore MG, Nathan CA, Orloff LA, O'Neill JP, Parker N. Safety Recommendations for Evaluation and Surgery of the Head and Neck during the COVID-19 Pandemic. JAMA Otolaryngology Head \& Neck Surgery. 2020 Mar 29.

19. Updated Intercollegiate General Surgery Guidance on COVID-19 available at https://www.rcseng. ac.uk/coronavirus/joint-guidance-for-surgeons-v2/( (Accessed on 24 ${ }^{\text {th }}$ April,2020)

20. Li B, Yang J, Zhao F, Zhi L, Wang X, Liu L, Bi Z, Zhao Y. Prevalence and impact of cardiovascular metabolic diseases on COVID-19 in China. Clinical Research in Cardiology. 2020 Mar 11:1-8.

21. Portnoy J, Waller M, Elliott T. Telemedicine in the Era of COVID-19 [published online ahead of print, 2020 Mar 24]. J Allergy Clin Immunol Pract. 2020;. doi:10.1016/j.jaip.2020.03.008

22. Day AT, Sher DJ, Lee RC, Truelson JM, Myers LL, Sumer BD, Stankova L, Tillman BN, Hughes RS, Khan SA, Gordin EA. Head and neck oncology during the COVID-19 pandemic: Reconsidering traditional treatment paradigms in light of new surgical and other multilevel risks. Oral Oncology. 2020 Apr 6:104684.

23. Simcock R, Thomas TV, Mercy CE, Filippi AR, Katz MA, Pereira IJ, Saeed H. COVID-19: Global radiation oncology's targeted response for pandemic preparedness. Clinical and Translational Radiation Oncology. 2020 Mar 24.

24. Schultz P, Morvan JB, Fakhry N, Moriniere S, Vergez S, Lacroix C, Bartier S, Barry B, Babin E, Couloigner V, Atallah I. French consensus regarding precautions during tracheostomy and posttracheostomy care in the context of COVID-19 pandemic. European Annals of Otorhinolaryngology, Head and Neck Diseases. 2020 Apr 9

25. Novara G, Giannarini G, De Nunzio C, Porpiglia F, Ficarra V. Risk of SARS-CoV-2 Diffusion when Performing Minimally Invasive Surgery During the COVID-19 Pandemic. European Urology. 2020 Apr 13. 